Military Technical College Kobry El-Kobbah, Cairo, Egypt

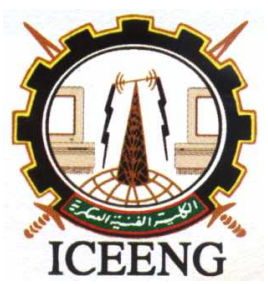

\author{
$6^{\text {th }}$ International Conference \\ on Electrical Engineering \\ ICEENG 2008
}

\title{
Microcontroller based feedforward digital controller for laser frequency locking for wide band WDM communication systems
}

\author{
By \\ Samy Ghoniemy*
}

\section{Abstract:}

In this paper, feed forward controller model based on the laser's inverse model is proposed. An enhanced digital control algorithm for 3rd, 5th, and 7th and possibly higher harmonics for laser frequency locking is developed. A generic diagram for the digital implementation of the proposed controller is presented. Digital implementation of the control algorithm modules is proposed and discussed. Hardware realization based on microcontrollers for the proposed digital controller is introduced and described for $\mathrm{He}-\mathrm{Ne}$ laser systems. Simulation results of the full system showed that using higher harmonics in laser frequency locking yields better performance in regard to the frequency shifts, improved noise performance, and hence better reproducibility. It also showed that the frequency difference between lasers locked with higher order harmonic locking using the digital realization is better than that locked with lower order harmonic locking. The measurement results using the lab version of the realized controller and $\mathrm{He}-\mathrm{Ne}$ laser system is presented and found in a very good agreement with the simulation results which assure that the proposed digital controller can be accurately used for transmitting multiple users' data over WDM communication systems with acceptable stability.

\section{Keywords:}

Frequency stabilization, frequency-locking, feedforward digital controller 


\section{Introduction:}

A wide range of scientific and application areas of research including frequently stabilization [1], CW frequency conversion [2], optical frequency standards [3], wide band frequency wave division multiplexed communication links [4], antenna remoting and laser frequency stabilization, require frequency locking of laser to optical cavities.

Many experiments and practical applications require a well-defined frequency, but over time, the central frequency will drift causing effective nonlinearity in the system. This procedure typically requires the generation of frequency error signal that is proportional to the difference between the laser frequency and the cavity resonance, which passes through zero at the lock frequency.

Wavelength modulation spectroscopic techniques are sometimes used to stabilize the frequency of a laser used in applications in differential absorption LIDAR, fiber optic communications, and interferometry [5-7]. The requirements for frequency stability.

A useful technique for controlling the wavelength of the pulsed laser transmitter is to inject seed with a tunable $\mathrm{CW}$ laser. By controlling the wavelength of the $\mathrm{CW}$ laser, in this case by actively locking it to an absorption line, spectral control and purity can be impressed on the pulsed laser. Stabilization must be held over the course of many hours during LIDAR measurements carried out in high-vibration environments such as on board an aircraft. Fiber optic communication systems using wavelength division multiplexing also require stable-wavelength lasers, but with today's standard channel spacing on the order of $10 \mathrm{GHz}$, the required level of stabilization can be obtained with relatively coarse calibration to a wavelength meter or e'talon. As channel spacing become smaller, a more precise wavelength reference may be required with performance similar to the system described here. The idea behind the wavelength locking is to generate a signal approximating the derivative of the absorption line shape with respect to wavelength by placing a small modulation on the laser. The interaction of the modulation sidebands with the absorption line creates the derivative-like signal, which has the useful property of going through a zero-crossing when the laser is on line center with a linear region around the line center, as shown in figure (1).

Locking is accomplished by implementing a feedback loop to actively tune the laser to keep the spectroscopic signal (now used as a control loop error signal) at the zero crossing. When the modulation frequency is large and the depth of modulation is small, the technique is referred to as frequency modulation spectroscopy. The nomenclature of wavelength modulation spectroscopy describes a case of small modulation frequency with a large depth of modulation. The resulting signals are similar, but in general the frequency modulation case enables a better SNR at the expense of more complicated electronic implementation compared to the wavelength modulation technique [8]. In the investigation described here, the wavelength modulation technique is used, but the 
application could be adapted to frequency modulation.

While frequency locking can be achieved to within a small range around line center, it suffers from a flaw in that the zero signal corresponding to line center can be confused with the zero signal seen when the laser wavelength is far from line center. For the frequency locking to work, the laser must first be manually tuned close to line center, within the locking range identified in figure (1), to make sure the locking circuit is operating on the zero corresponding to line center. The lock can then be activated to engage the feedback loop. But if the experiment is shut down or encounters a problem, the manual tuning must be repeated. This manual tuning requires skilled operation and extra equipment, such as observing the wavelength on a wavemeter or by stepping through the laser tuning and watching an oscilloscope for a signal indicative of being within the locking range. This issue is addressed in [9] for a He-Ne laser stabilized to an intracavity iodine cell with a digital control system that automatically mapped out the spectral positions of iodine absorption lines. The work reported here includes use with various types of lasers to external reference sources.

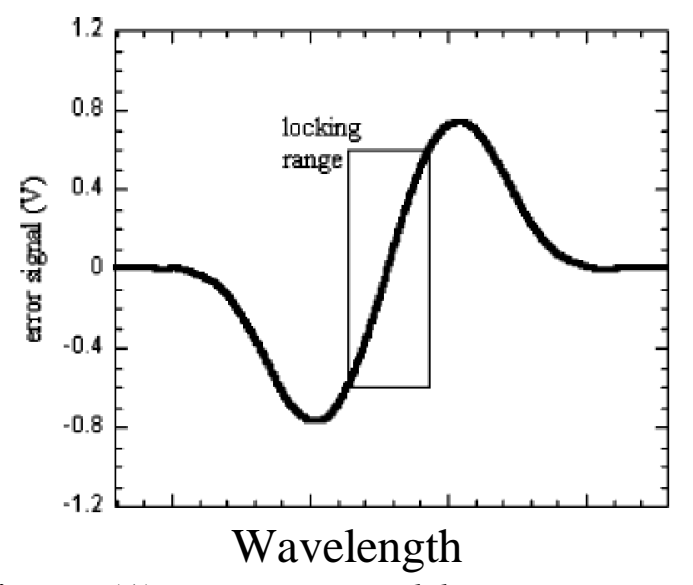

Figure (1): Derivative-like error signal

In this paper, we propose a digital signal processing based digital control system using inexpensive microcontrollers for laser frequency over a wide frequency band and can be integrated with most of the laser systems. To demonstrate this ability the proposed system is used with He-Ne laser system.

\section{General system configuration:}

The general block diagram of the digital laser frequency locking system is shown in Figure (2). The laser in this design could be any single-frequency source with a mechanism for tuning, including a distributed feedback diode laser with current tuning, a external cavity diode laser with piezoelectric translator (PZT) tuning, or a solid state laser tuned by PZT movement of the output coupler. Several examples of frequency locking various laser types using the wavelength modulation technique have been 
published with a typical result that stabilization can be maintained to within a few percent of the linewidth of the reference absorption feature [10-12]. For a Dopplerbroadened absorption line of typically $1 \mathrm{GHz}$ width, this translates into locking within $10 \mathrm{MHz}$ of absorption line center.

The idea behind this automated frequency stabilization process is to ramp the laser frequency until it enters the frequency-locking zone. Once inside the locking zone, the frequency ramp is halted and the locking control loop is activated. This transition from ramping to locking is indicated in Figure (2) by a two-position switch that is electronically addressed using the microcontroller. Detection of the frequency locking zone is done by taking the derivative (with respect to time) of the error signal during the frequency ramp. The derivative of the error signal peaks when the laser approaches line center, and has a useful property of swinging to positive polarity when it enters the frequency locking zone. A comparator is triggered on seeing a positive polarity of the derivative, which subsequently triggers a digital circuit to shut off the frequency ramp and activate the feedback loop for locking onto the zero-crossing. The feedback loop is implemented here by a proportional-integral-differential (PID) controller. The PID used is adapted from a commercial off-the-shelf component commonly used in industrial process control and digitally programmed and downloaded in the microcontroller's EPROM.

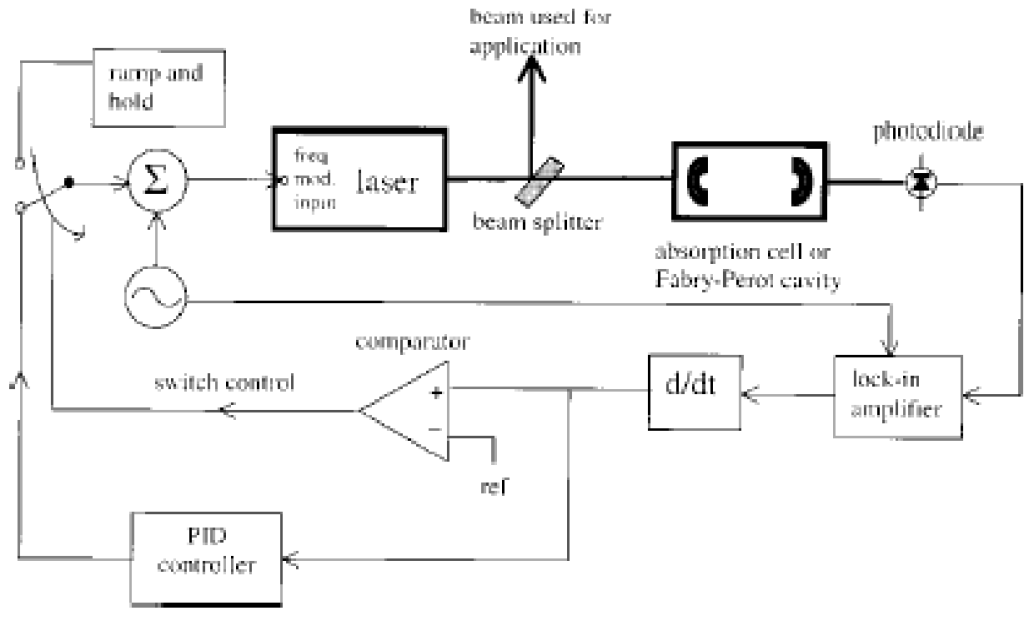

Figure (2): General block diagram of the digital laser frequency locking system.

\section{Operational system components:}

\section{A. Digital control design}

The operational block diagram of the digital controller and the digital control algorithm are shown in Figures (3) and (4). Figure (3), shows the main components of the control system in which the analog to digital A/D and digital to analog D/A converters are connected to a PIC microcontroller $(\mu \mathrm{C})$ which performs the control algorithm. The 
microcontroller is controlled and monitored by a general purpose PC computer. The computer loads the program into the $\mu \mathrm{C}$ and sends a command that makes the $\mu \mathrm{C}$ lock the laser, or change its parameters. The PC can also read information from the $\mu \mathrm{C}$, such as the output of the harmonic detectors or the tuning voltage. Some user controls and displays are attached to the microcontroller so that the unit can be operated stand-alone. The personal computer for monitoring of the detector outputs or for full remote operation. The microcontroller consists of 16 bit microprocessors with the BASIC interpreter, analog-to-digital converters $\mathrm{A} / \mathrm{D}$, counters, pulse-width modulators $\mathrm{PWMs}$ and digital input/output D I/O.

The program for frequency stabilization is loaded into the microcontroller through a parallel interface of the PC. The PC receives various information such as laser power, beat frequency, and error signal through a serial interface. It should be noted that the proposed laser controller in this paper is operated stand alone without the PC after the optimum control parameters are determined. The laser beam from the rear mirror is divided into two orthogonal linearly polarized components by the PBS. These beams are received by two photodiodes (PD1, PD2). One of the signals from the photodiode, PD1, is amplified and converted to digital signals by the 16 bit $\mathrm{A} / \mathrm{D}$ converter of the $\mu \mathrm{C}$. The $\mu \mathrm{C}$ compares the received digital signal with a reference digital signal and obtains the power difference between them using customized stored software. This difference is used as an error signal for control. The error signal from the power difference or beat frequency is amplified, accumulated and differentiated by software implemented (PID). The PWM provides the correction signal to the taps of the automatic gain amplifiers in the forward path. The duty ratio of the 10 bit PWM is proportional to the error signal through the PID algorithm.

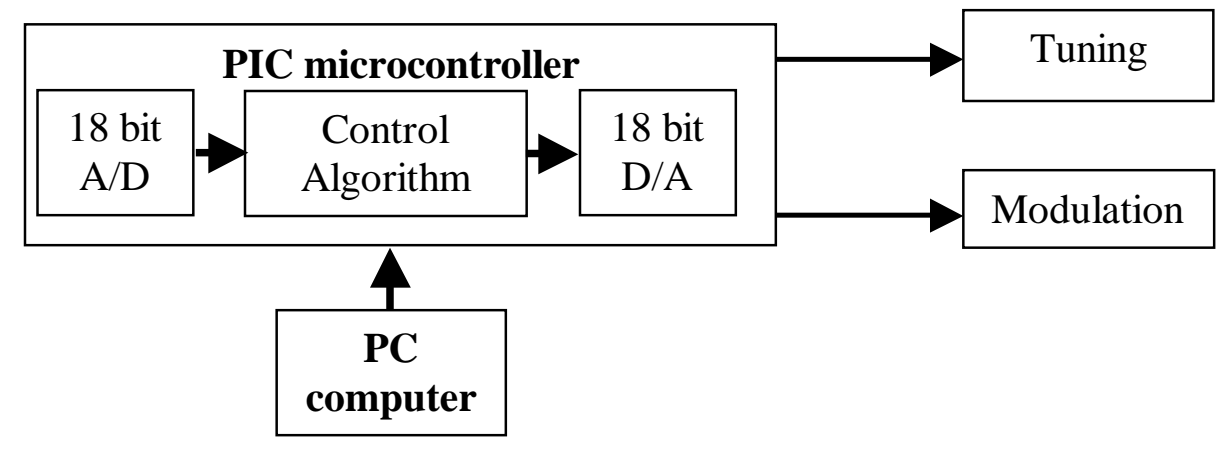

Figure (3): General block diagram of the digital laser frequency locking system.

The most critical components in this kind of digital control system are the A/D and D/A converters. High quality 18-bit converters have been chosen. One input is required for the control system. The sampling frequency is $52083 \mathrm{~Hz}$. This limits the input bandwidth and thus the maximum usable modulation frequency. There are two outputs. One output is used for the sine wave modulation signal and one for tuning the laser. It is 
very important that the harmonic distortion in the modulation signal and the input $A / D$ converter is low. If this were not the case, offsets in the locking might occur. The tuning voltage output from the D/A converter is amplified by a low noise high voltage amplifier designed specifically for the system. The tuning range is $600 \mathrm{~V}$ in a balanced configuration.

\section{A. Digital controller design}

Figure (3), shows the behavioral block diagram of the control algorithm that performs the control loop. The harmonic detection is based on a multiplier. The system has two detectors. One always detects the third harmonic by multiplying the input signal with a reference sinusoidal signal three times higher in frequency than the modulation signal. The phase of this sinusoidal signal is locked to the modulation signal and the phase difference between these can be adjusted. The other detector is similar to the third harmonic detector with the only exception that it detects higher harmonics (5th, 7th, ...etc). The sine wave generator is implemented by a lookup table. The outputs of the detectors are low pass filtered to remove some of the noise. The filtered detector signals are fed to the PI controller through a switch network that allows different locking configurations. Furthermore, there are additional digital low-pass filters at the detector outputs which filter the monitoring signals.

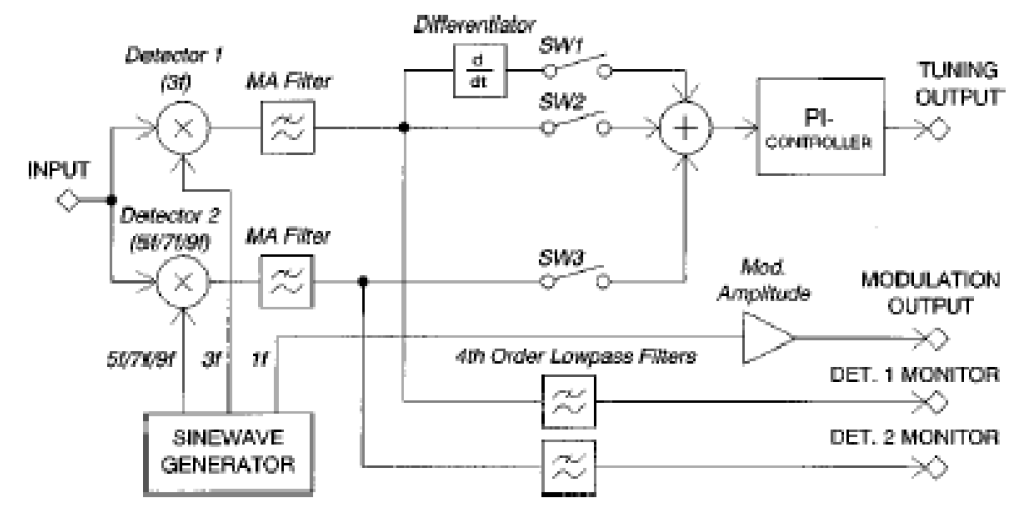

Figure (4): General block diagram of the digital controller.

\section{B. Digital control algorithm}

The control program was written with BASIC language and was loaded into the $\mu \mathrm{C}$ through a parallel interface. The data from the $\mu \mathrm{C}$, such as the error signal are displayed the PC monitor. Real-time communication is very useful for finding the optimum values of the control parameters. The $\mu \mathrm{C}$ can perform approximately 40,000 instructions per second. The control speed (update rate of the error signal) depends on the control algorithm. In this case a maximum control speed of about $3 \mathrm{~Hz}$ is used for a $30 \%$ of the period ( 1 second), 20 data sets from A/D converters are averaged to reduce the noise of the analog acquisition are averaged to reduce the noise of the analog acquisition system 
detecting the laser power difference between the two modes. The control speed is limited because the high control speed decreases the dynamic range of the counter.

Figure 4 shows the outline of the digital algorithm that performs the control loop. The harmonic detection is based on a multiplier. The system has two detectors. One always detects the third harmonic by multiplying the input signal with a sinusoidal signal three times higher in frequency than the modulation signal. The phase of this sinusoidal signal is locked to the modulation signal and the phase difference between these can be adjusted. The other detector is similar to the third harmonic detector with the only exception that it detects higher harmonics (5th, 7th, etc.). The sine wave generator is implemented by a lookup table. The outputs of the detectors are low pass filtered to remove some of the noise. The filtered detector signals are fed to the PI controller through a switch network that allows different locking configurations. The third harmonic output can optionally be differentiated which is used in the new $3 \mathrm{hD}$ locking technique that is described later. Furthermore, there are additional digital low-pass filters at the detector outputs which filter the monitoring signals.

A complete diagram for the overall control system is shown in Figure (5). A $3 \mathrm{hD}$ locking mode is assumed. In the figure The MA (moving average) filters with a rectangular shaped impulse response are simple digital low-pass filters. Even if they are not the best choice of low-pass filters in the general case, they have favorable zero positions for this application. In this figure, a recursive structure has been chosen to increase computing efficiency. The filter transfer function (where $\mathrm{z}$ is the Z-transform variable) is the following:.

$$
H(z)=\frac{1-Z^{N}}{1+Z^{1}}=\sum_{N-0}^{N-1} Z^{-i}
$$

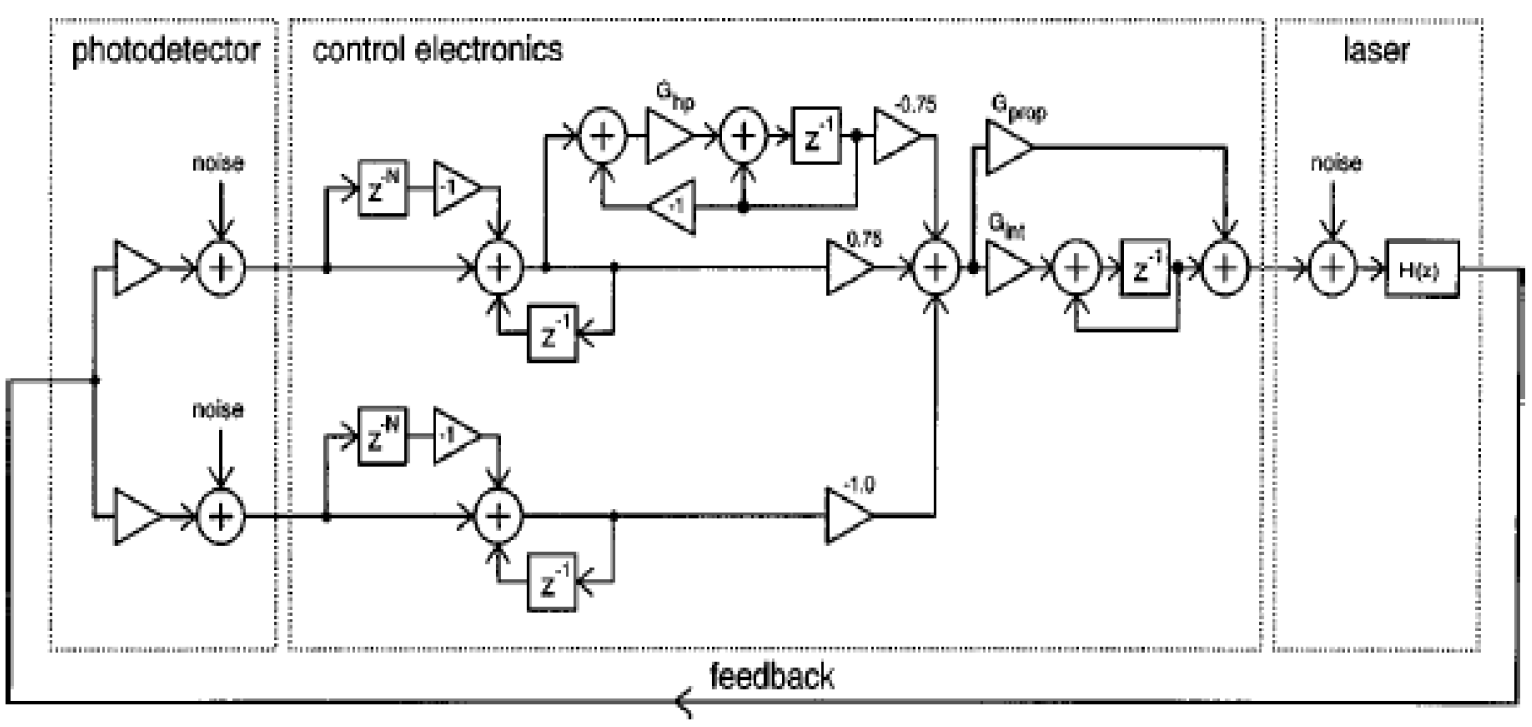

Figure (5): Developed digital controller. 


$$
H(z)=\frac{1-Z^{N}}{1+Z^{1}}=\sum_{N-0}^{N-1} Z^{-i}
$$

The PI controller circuit is a simple integrator (accumulator) with a bypass path. Integration gain and proportional gain can be adjusted separately. The so-called time constant is related to the ratio of proportional and integration gain. The integration gain is kept constant at 6 while the proportional gain increases from 4 to 8 in the different curves. The actual gain (Gprop ,Gint) increases $6 \mathrm{~dB}$ for each increment of one in the integer gain parameter ( $\mathrm{i}$ gain, $\mathrm{p}$ gain). The transfer function of the PI controller is given in (2). Figure (6) shows the spectrum of frequencies the input is sensitive to. The used modulation frequency is $2848 \mathrm{~Hz}$. At the fifth harmonic $(14241 \mathrm{~Hz})$ the response of the system is infinite due to the integration. Close to the third harmonic $(8545 \mathrm{~Hz})$ the gain is also high, but exactly at the third harmonic there is a zero in the response curve.

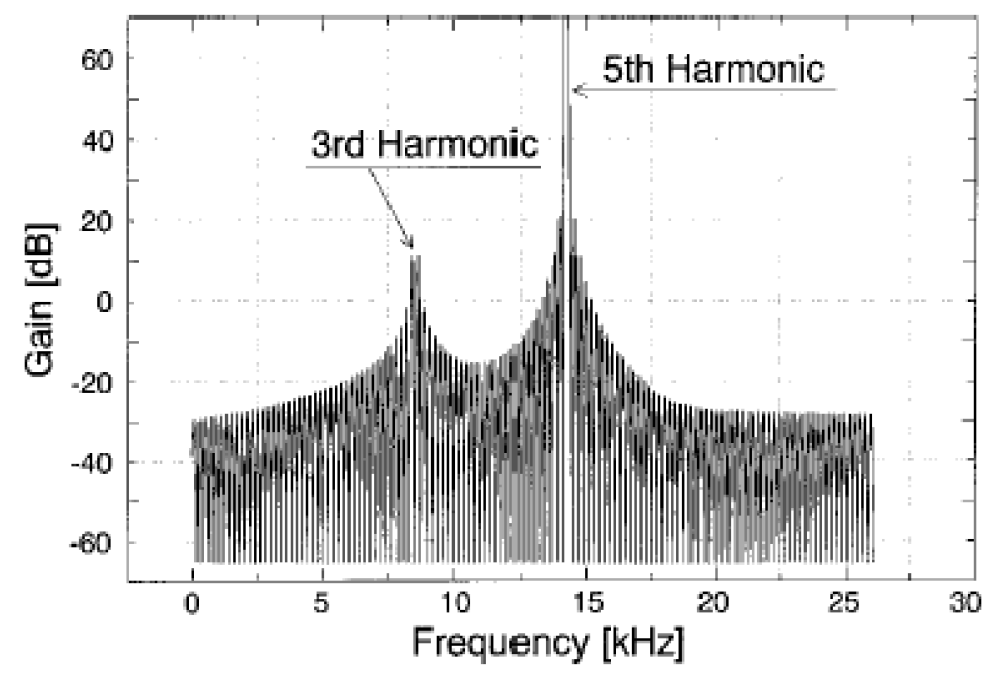

Figure (6): Open loop gain function spectrum seen from input domain.

The identification of the absorption lines in the system is based on a simple form of a matched filter. The laser is first swept over a wide range. The produced sequence of the third harmonic detector output is then passed through this filter. The maximum of the filter output identifies the position of the absorption lines. Further algorithms take care of finding the right absorption peak. Hysterics of the piezoelectric transducers and temperature drift had to be compensated for in the search algorithm. Figure (7) shows the frequency sweep and the resulted output at the matched filter. 


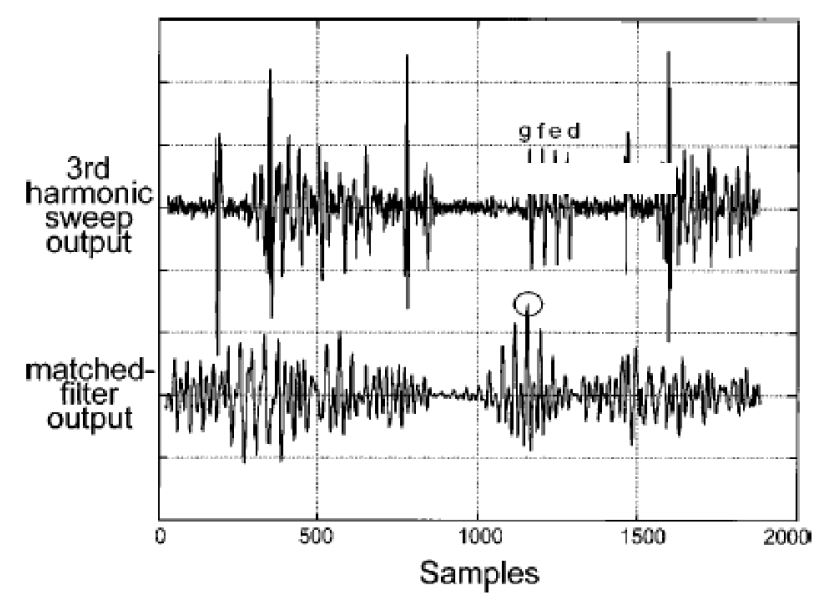

Figure (7): Laser frequency sweep and the resulted output at the matched filter. The maximum of the matched filter output indicates the start of the group of absorption lines.

\section{Results and analysis:}

The hardware system has not been tested however it works reliably in simulations. The control algorithm is basically a simple PID controller but is greatly enhanced by the $3 \mathrm{hD}$ locking method. Implementing into the system an enhanced digital controller algorithm that uses optimal filtering may improve the noise performance. This upgrade is easy to perform by just modifying software. However, the computing capacity of the system (40000of instructions per second) limits the complexity of the algorithm. The possibility to use higher harmonics in the locking has been very useful.

The frequency of a laser locked with third harmonic locking was compared with a laser locked with third, fifth and seventh harmonic locking using the digital system. The frequency differences were as follows: third harmonic: $10.863 .1 \mathrm{kHz}$, fifth harmonic: $38.964 .0 \mathrm{kHz}$, and seventh harmonic: $58.968 .9 \mathrm{kHz}$. The uncertainty will increase with higher order harmonics because the strength of the harmonic will reduce proportional to the order. Thus the laser stability will be degraded. A beat frequency measurement between the digital system and an analog system was performed. The resulted Allan variance is plotted in Figure (8). The data were obtained by measuring the beat signal between our $\mathrm{He}-\mathrm{Ne}$ laser detected output and a reference stabilized sinusoidal. The discrepancy in the results is caused by the smaller dynamic range of the frequency counting system. Our results on the frequency stability are similar to those reported by others. However, the frequency stability of our lasers at the short period is better than that required as a light source of the normal length measuring interferometer. 


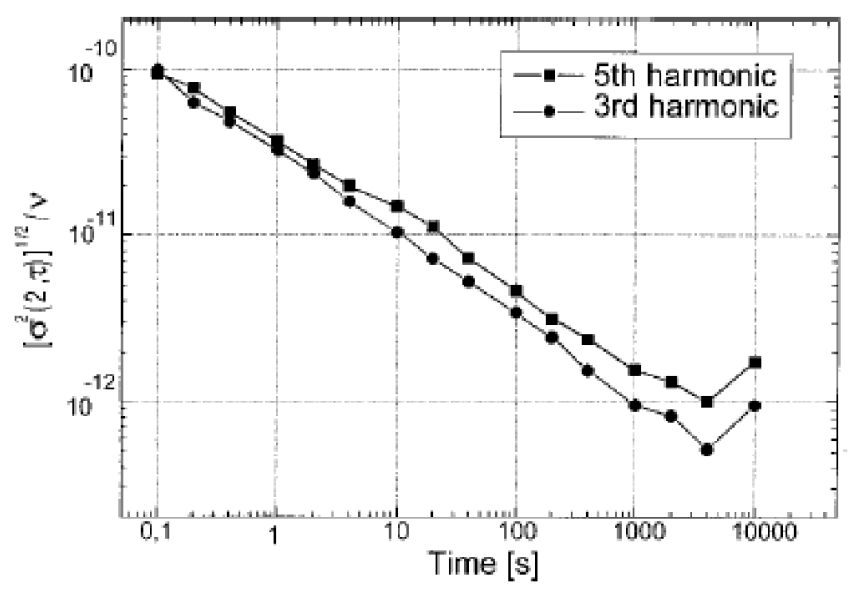

Figure (8): Allan variance of a beat frequency measurement of the digital system locked with the third and fifth harmonic method

Figure (8) showed that the stability of the fifth harmonic locking is slightly degraded at longer gate times because of the weak fifth harmonic signal.

\section{Conclusions:}

The frequency stabilization of $633 \mathrm{~nm} \mathrm{He}-\mathrm{Ne}$ lasers is performed by a digital control system. The microcontroller used in the system has various I/O functions such as A/D converter, counter, pulse-width modulator, and digital input/output. In spite of the additional expense of the microcontroller (about \$10), our digital control system has many advantages compared to the analog system. The control circuit can be very compactly designed and it can also be applied at a variety of frequency stabilization schemes without modification of the electronic circuit. By real-time communication and system modification by software, the optimum values of several control parameters could easily be determined. Program written and debugged using the BASIC interpreter. Though the processing speed of the microprocessor with the BASIC interpreter is low, so, it can be applied to the frequency stabilization of an internal mirror He-Ne laser because the laser cavity length is usually controlled by thermal means with low response time. However, the proposed design is practical and powerful for the length measuring interferometer requiring an optical frequency standard with an accuracy of $10^{-8}$. This system has a possibility for further improvements by incorporation of a thin-film heater with fast response time.

\section{References:}

[1] F. Bondu, P. Fritschel, C. N. Mann and A. Brillet, "Ultrahigh-spectral-purity laser for the VIRGO experiment," Opt. Lett., Vol 21, 8, 582-584 (1996) 
[2] K. Schneider, S. Schiller, J. Mlynek, M. Bode and I. Freitag, "1.1-W singlefrequency 532-nm radiation by second-harmonic generation of miniature Nd:YAG ring laser," Opt. Lett. Vol 21, 1999 (1996)

[3] B.C. Young, F.C. Cruz, W.M. Itano, J.C. Bergquist, (Visible lasers with sub hertz linewidths," Phys. Rev. Lett., Vol 82, 19, 3799-3802, (1999)

[4] A. Ratnapala et al, " Laser frequency locking by direct measurement of detuning," Optics Letters, Vol. 29, No. 23-December 1, 2004.

[5] Ikegami, S. Sudo, and Y. Sakai, Frequency Stabilization of Semiconductor Laser Diodes, Artech House, Norwood, MA, 1995.

[6] W. C. Edwards, L. P. Petway, and C. W. Antill, "Performance improvements to the lidar atmospheric sensing experiment (LASE),' in Proc. 19th Int. Laser Radar Conf., NASA/CP-1998-20761 pp. 815- 817, 1998.

[7] D. Bruneau, T. Arnaud des Lions, P. Quaglia, and J. Pelon, “Injection seeded pulsed alexandrite laser for differential absorption LIDAR application," Appl. Opt. 33, 3941-3950 1994.

[8] J. A. Silver, "Frequency-modulation spectroscopy for trace species detection: theory and comparison among experimental methods,' Appl. Opt. 31, 707-717, 1992.

[9] T. Ahola, J. Hu, and E. Ikonen, "A digital control system for the iodine stabilized He-Ne laser,' Rev. Sci. Instrum. 69, 1934-1937, 1998.

[10] G. J. Koch, A. L. Cook, C. M. Fitzgerald, and A. N. Dharamsi, "Frequency stabilization of a diode laser to absorption lines of water vapor in 944-nm wavelength region,', Opt. Eng. 40, 525-528, 2001.

[11] C. M. Fitzgerald, G. J. Koch, A. M. Bullock, and A. N. Dharamsi, "Wavelength modulation spectroscopy of water vapor and laser line center stabilization at 1.462 mm for lidar applications,' ' Proc. SPIE 3945, 98-105 2000!.

[12] G. J. Koch, A. N. Dharamsi, C. M. Fitzgerald, and J. C. McCarthy, "Frequency stabilization of a Ho:Tm:YLF laser to absorption lines of carbon dioxide,' Appl. Opt. 39, 3664-3669, 2000. 